\title{
NEOCONSTITUCIONALISMO Y ESPECIFICIDAD DE LA INTERPRETACIÓN CONSTITUCIONAL
}

\begin{abstract}
$\mathrm{n}$ qué consiste, si es que se da, la especificidad de la interpretación constitucional? ¿Qué distingue la interpretación de la constitución de la interpretación de cualquier texto normativo? ¿La interpretación de la constitución, es algo distinto de la interpretación de la ley? Se pueden leer cada vez con mayor frecuencia argumentaciones dirigidas a sostener la especificidad de la interpretación constitucional respecto a la de otros documentos normativos $y$, específicamente, respecto a la ley. La hipótesis de la que parte mi reflexión es que la especificidad de la interpretación constitucional, si se da, está estrechamente ligada a una peculiar configuración del objeto «constitución».
\end{abstract}

\section{Neoconstitucionalismo}

Si bien es cierto que la tesis sobre la especificidad de la interpretación constitucional encuentra partidarios en diversas disciplinas, en el ámbito de la filosofía del derecho viene defendida, en particular, por un grupo de iusfilósofos que comparten un peculiar modo de acercarse al derecho. He llamado a tal corriente de pensamiento neoconstitucionalismo. Me refiero, en particular, a autores como Ronald Dworkin ${ }^{1}$, Robert Alexy², Gustavo Zagrebelsky ${ }^{3}$ y, sólo en parte, Carlos S. Nino ${ }^{4}$. Probablemente estos iusfilósofos no se reconocen dentro de un movimiento unitario, pero, en favor de mi tesis, en sus argumentaciones es posible encontrar el uso de algunas nociones peculiares que posibilita que sean agrupados dentro de una única corriente iusfilosófica. Estas características se pueden sintetizar en algunas formulaciones:

${ }^{1}$ Cfr. R. Dworkin, Freedom's Law, Oxford UP, Oxford, 1996.

${ }^{2}$ Cfr. R. Alexy, El concepto y la validez del derecho, Gedisa, Barcelona, 1994.

${ }^{3}$ Cfr. G. Zagrebelsky, Il diritto mite, Einaudi, Torino, 1992; trad. esp. El derecho dúctil.

${ }^{4}$ Cfr. C. S. Nino, The Constitution of Deliberative Democracy, Yale UP, New Haven London, 1996. 
1) Principios versus normas. Con esta formulación me refiero a la expresión de tesis dirigidas a sostener que el ordenamiento jurídico no se compone sólo de normas, sino de normas y principios. Los principios se diferencian de las normas por algunas características: la dimensión de peso o de importancia y la no aplicabilidad del llamado modo del «todo o nada», según el cual son aplicables al caso concreto sólo prima facie. Distintas son las conceptualizaciones de estos estándars normativos, pero, en general, son vistos como valores morales positivizados. Respecto al texto constitucional, aunque los principios no sean una peculiaridad absoluta del mismo, sí que es cierto que en él, en general, y en la mayor parte de las constituciones contemporáneas, se encuentran presentes en un número particularmente elevado, expresados en un lenguaje extremadamente vago y con un alto nivel de abstracción, hasta determinar un cambio cualitativo del propio documento. Junto a la tesis de los principios se encuentra, normalmente, la expresión de una tesis prescriptiva según la cual los jueces deberían servirse en mayor medida de tales estándares en la interpretación y en la argumentación jurídica. Eso vale, en primer lugar, como recomendación dirigida al juez constitucional, para el que se sugiere el uso de justificaciones basadas en argumentos de justicia. Pero, más en general, y también para ordenamientos en los que se cuenta con un órgano concentrado para el juicio de constitucionalidad de las leyes, esta tesis tiende a sugerir una interpretación de la constitución y la consiguiente argumentación, dirigida a extraer del texto constitucional normas y principios idóneos para regular directamente las controversias.

2) Ponderación versus subsunción. Con esta expresión me refiero al peculiar método interpretativo/aplicativo que requieren los principios. En efecto, ligada a la tesis de los principios se encuentra una peculiar teoría de la interpretación. Los principios no serían interpretables/aplicables según el común método llamado «subsunción», peculiar de las normas. Los principios necesitarían un método llamado ponderación o balanceo. Esta técnica interpretativa consta principalmente de tres pasos: a) en primer lugar, una operación interpretativa tendente a localizar los principios aplicables al caso concreto. Se presupone que los principios idóneos para regular el caso concreto son siempre más de uno y que se presentan siempre en una relación de antinomia parcial. Es decir, se presupone que ambos regulan el caso, pero que tales regulaciones son sólo parcialmente coincidentes; b) en segundo lugar, los principios así localizados son internamente sopesados para poder ponerlos en relación jerárquica, obviamente axiológica; el principio de mayor peso prevalece sobre el principio de menor peso; c) en tercer

\footnotetext{
${ }^{5}$ Cfr. R. Guastini, Distinguendo, Giappichelli, Torino, 1996.
} 
lugar, la jerarquía axiológica instaurada por el intérprete se caracteriza por no estar construida en abstracto, sino en concreto. $\mathrm{O}$ sea, se construye en relación al caso examinado y tiende, por tanto, a presentarse cambiante en el sentido de que dependerá de las características peculiares de cada caso específico. De este modo, el principio que prevalece en el caso examinado podrá sucesivamente ceder en el juicio de otro caso concreto. La relación axiológica, pues, se da cada vez para cada caso individual en base a los juicios de valor formulados por el juez individual.

3) Constitución versus independencia del legislador. Con esta formulación me refiero a la expresión de una tesis dirigida a sostener una penetración general del texto constitucional. Si la subordinación de la ley puede parecer obvia desde el momento que se afirma la superioridad del texto constitucional, lo que es peculiar de este modo de ver las cosas es la «materialización»o «sustancialización» de la constitución ${ }^{6}$. Con el uso de estos términos se quiere indicar que la constitución no tiene por objeto únicamente la distribución y la organización de los poderes, sino que presenta un contenido sustancial que condiciona la validez de las normas subconstitucionales. Esto, no ya en el sentido kelseniano según el cual la constitución constituya una suerte de «marco» en el cual el legislador ordinario lleva a cabo sus decisiones políticas?. Sin embargo, el «contenido sustancial condicionante» cumple una función muy parecida a la que previamente había desarrollado el derecho natural. Derecho natural, primero, derecho constitucional, ahora, derecho, en todo caso, que el legislador debe necesariamente considerar como una guía para la producción legislativa al que debe, por tanto, adecuarse y desarrollar. La ley, pues, cede el paso a la constitución y se convierte en un instrumento de mediación entre exigencias prácticas y exigencias constitucionales.

4) Jueces versus libertad del legislador. Con esta formulación me refiero a la expresión de una tesis favorable a la interpretación creativa de la jurisprudencia. Al juez, supuesta la presencia de los principios, el consiguiente abandono del método de la subsunción, la penetración general del texto constitucional, se le encarga un continuo adecuamiento de la legislación a las prescripciones constitucionales. La sustancialización de la constitución sitúa en primer plano la consideración de las exigencias de justicia sustancial que el caso concreto trae consigo. Por lo cual, si con anterioridad el juez interpretaba/aplicaba el derecho independientemente de la valora-

${ }^{6}$ Cfr. M. La Torre, Derecho y conceptos de derecho. Tendencias evolutivas desde una perspectiva europea, en «Revista del Centro de Estudios Constitucionales», 16, 1993, pp. 67-93.

${ }^{7}$ Cfr. H. Kelsen, Sulla teoria dell'interpretazione (1934), en P. Comanducci y R. Guastini (a cura di), L'analisi del ragionamento giuridico, cit., pp. 107-120, p. 108. 
ción del caso concreto, ahora el juez debe interpretar el derecho a la luz de las exigencias de justicia vehiculadas por el caso. La interpretación moral del caso incide, de este modo, en la interpretación de las palabras del derecho. Debido a este peculiar papel asumido por el juez, éste tiende a configurarse como un fundamental elemento racionalizador del sistema jurídico.

\section{Modelo axiológico de la constitución concebida como norma, neoconstitucionalismo y anti-iuspositivismo}

Aquello que distingue la doctrina neoconstitucionalista es la adopción de un peculiar modelo constitucional. Tal modelo ha sido denominado «modelo axiológico de la constitución concebida como norma» ${ }^{8}$. «Constitución» designa aquel conjunto de reglas jurídicas positivas, fundamentales respecto a las demás reglas del conjunto, pero que pasan a ser «constitución» en razón del particular contenido que expresan, y no en razón del sujeto que ha redactado el documento. La adopción de este modelo constitucional se conjuga con el interés principal de la doctrina neoconstitucionalista. En efecto, ésta se mueva principalmente en la perspectiva de la justificación, a un nivel de lenguaje en el que se considera central la noción de razón para actuar. Una razón para actuar es una razón que justifica un comportamiento. Se sostiene que no es posible comprender el funcionamiento jurídico sin ponerlo en relación con la noción de razón, sólo de esta forma se clarifica la dimensión normativa del derecho. Si el primitivo modelo iuspositivista identificaba la razón para la acción en la orden del soberano ${ }^{9}$, el modelo evolucionado de iuspositivismo rechaza tal planteamiento -que, en definitiva, identificaba una «razón justificativa» aludiendo a un hecho, cometiendo así una aporía lógica- y afirma que la justificación de una orden (o de una norma) reside necesariamente en otra norma, y no en el hecho de que alguien, quien quiera que sea, haya ordenado algo. Así, pues, la observancia de la constitución, en la medida en que ésta sea también una norma, debe justificarse en base a normas de carácter superior. Dicho de otro modo, si un juez recorre la cadena de competencia, partiendo de la norma aplicable al caso concreto, llegará a la carta constitucional. Pero esta última, si bien representa un límite interno al ordenamiento jurídico, contextualmente constituye un puente que permite el pasaje al discurso moral. Este último es, en última instancia, el único discurso que puede proveer una justificación a la observancia o a la aplicación del derecho. En efecto, una norma

${ }^{8}$ Cfr. M. Dogliani, Introduzione al diritto costituzionale, Il Mulino, Bologna, 1994; P. Comanducci, Interpretazioni della costituzione, manuscrito inédito.

${ }^{9}$ Cfr. J. Austin, The Province of Jurisprudence Determined (1954); R. Shiner, Norm and Nature, Clarendon Press, Oxford, 1992. 
moral se acepta en virtud de su contenido y no por su génesis; ella sola, por tanto, constituye la justificación última de una acción o de una decisión. La justificación jurídica, pues, es en última instancia necesariamente moral ${ }^{10}$.

Este peculiar modo de acercarse al derecho puede ser leído como un intento de revisión de la doctrina iuspositivista. En particular, esta revisión se refiere a la llamada «regla de reconocimiento». La conceptualización de los principios como normas morales positivizadas conduce a sostener que la regla de reconocimiento iuspositivista -regla que, como es sabido, se identifica sobre la base de un análisis de la génesis de las normas del ordenamiento- no está en condiciones de dar cuenta de estos estándares normativos o, mejor dicho, está en condiciones de reconocer sólo algunos de ellos. Los principios son, empero, fundamentales a nivel de justificación: permiten el paso del discurso jurídico al moral proveyendo, en última instancia, la justificación última de las decisiones jurídicas. Es preciso, pues, modificar la regla de reconocimiento y aceptar el hecho de que, en ciertos casos, y dado que los principios son jurídicos, el derecho es considerado válido no en razón de un test de pedigree sino en razón de un test moral, el cual, por definición, no se basa en la génesis de los principios sino en su contenido ${ }^{11}$.

Esto lleva a sostener, a distintos niveles ${ }^{12}$, la conexión necesaria entre derecho y moral. La conexión se verifica a nivel interpretativo. Al atribuir significado a los estándares denominados «principios», el operador debe primero atribuir significado a los valores morales subyacentes; es preciso, pues, que ofrezca una lectura moral de los mismos (y no literal). Desde el punto de vista del mero análisis lingüístico, los principios no tendrían nada que decir, perderían su valor prescriptivo; por el contrario, se comprenden a través de su ethos. El producto de la interpretación, al final de varios pasos valorativos requeridos, es una norma que pueda justificar una decisión en cuanto presenta un origen moral. La conexión entre derecho y moral se verifica, pues, también a nivel justificativo, ya que sólo mediante esa vía la decisión jurídica podría justificarse. A partir de ahí, la doctrina del neoconstitucionalismo sostiene la prevalencia del punto de vista interno, del punto de vista del participante, en relación con el del observador desinteresado. Sólo desde la perspectiva del participante es identificable la decisión

${ }^{10}$ Para la distinción entre justificación moral y justificación jurídica: cfr. P. Comanducci, Diritto, morale e politica, manuscrito inédito; J. J. Moreso, P. E. Navarro, M. C. Redondo, Argumentación jurídica, lógica y decisión judicial, en «Doxa», 11, 1992, pp. 247-262.

${ }^{11}$ En realidad se ha mostrado que esta interpretación de la regla de reconocimiento hartiana es parcial. Hart, en efecto, no limita la capacidad de reconocimiento de la regla sólo al derecho legislativo, sino que toma en cuenta también el derecho de fuente jurisprudencial. Cfr. W. J. Waluchow, Inclusive Legal Positivism, Clarendon Press, Oxford, cap. 8.

${ }^{12}$ Cfr. C. S. Nino, Derecho, moral y politica, Ariel, Barcelona, 1994. 
justificada. El observador, sin compartir el sustrato moral de los participantes, no podría identificar la efectiva justificación del derecho. Tendería a proveer una descripción falaz, puesto que remontándose de la norma en cuestión hasta la norma que la justifica y así sucesivamente construyendo la cadena de competencia, llegará hasta la última norma positiva del sistema, por ejemplo, a la constitución, pero más allá de este punto no estará en condiciones de identificar normas válidas, sino sólo la prescripción de alguna autoridad, por ejemplo, la asamblea constituyente, y considerará, entonces, que la justificación de las decisiones reside, en última instancia, en los hechos que han llevado a la existencia de las normas aplicadas. Pero, como se ha dicho, la justificación de una norma reside siempre en otra norma y no en los hechos en los que ha tenido su origen. Por tanto, en definitiva, el observador no podrá identificar la «real» justificación de las decisiones jurídicas.

Siguiendo esta línea doctrinal, también la ciencia jurídica debe asumir responsabilidades morales ${ }^{13}$. No puede limitarse a una descripción del derecho. Si hiciera esto último, en efecto, no sería de ninguna utilidad para la práctica jurídica, ya que no podría proveer un esquema conceptual de justificación de las decisiones. La doctrina neoconstitucionalista rechaza, por tanto, la adopción de un modelo descriptivo de la norma constitucional; modelo en el que «constitución» denota un conjunto de reglas jurídicas positivas consideradas respecto a las demás reglas, superiores o fundamentales. El rechazo del uso de este modelo se debe a su carácter descriptivo, y por eso mismo, incapaz de proveer razones para actuar. Si la ciencia tiene la tarea de reformular y reconstruir el ordenamiento jurídico, ayudando de este modo a los juristas prácticos a solucionar los problemas que surgen de la interpretación y la aplicación del derecho, debe usar conceptos normativos, conceptos que sin ser formulados en una perspectiva descriptiva tomen en consideración el contenido obligatorio del derecho.

\section{Neoconstitucionalismo y especificidad de la interpretación constitucional}

Para analizar el significado que asume la especificidad de la interpretación constitucional en el ámbito de la doctrina neoconstitucionalista haré referencia a los modelos constitucionales identificados anteriormente. De interpretación específica del texto constitucional se puede hablar en distintos sentidos ${ }^{14}$. Hay, en efecto, algunas características peculiares de la constitu-

${ }^{13}$ Cfr. C. S. Nino, Introduzione all'analisi del diritto (1980), Giappichelli, Torino, 1996; G. Zagrebelsky, Il diritto mite, cit.

${ }^{14}$ Cfr. R. Guastini, Specificità dell'interpretazione costituzionale?, en P. Comanducci y R. Guastini (a cura di), Analisi e diritto 1996, Giappichelli, Torino, 1996; L. Prieto Sanchís, Notas sobre la interpretación constitucional, en «Revista del Centro del Estudios Constitucionales», 9, 1991, pp. 175-198. 
ción que permiten hablar de especificidad. En primer lugar, se puede identificar una especificidad en base a los peculiares sujetos de la interpretación. En segundo lugar, se puede identificar el uso de particulares técnicas interpretativo/aplicativas adoptadas para el texto constitucional. En tercer lugar, pueden darse peculiares efectos de las sentencias del juez constitucional. En cuarto lugar, se puede hablar de especificidad de la interpretación constitucional en razón de la rigidez o de la flexibilidad de la constitución. En quinto lugar, se puede sostener la especificidad en base al objeto «constitución».

a) Peculiaridad del sujeto. La especificidad de la interpretación constitucional en base al sujeto de la interpretación depende de la estructura del ordenamiento ${ }^{15}$. Si, por ejemplo, el ordenamiento considerado prevé un juez constitucional concentrado en un órgano expreso se puede identificar una especificidad de la interpretación de la constitución respecto a la interpretación de la ley en cuanto a la competencia de la interpretación/aplicación de una y otra sea conferida por el ordenamiento a sujetos distintos. $\mathrm{Si}$ el ordenamiento considerado prevé, por el contrario, un control de constitucionalidad difuso, no habría especificidad de la interpretación constitucional respecto a la interpretación de la ley en cuanto que hay un único sujeto competente para la interpretación/aplicación de una y otra.

b) Efectos peculiares de las sentencias del juez constitucional. Unido al tipo de control de constitucionalidad previsto por el ordenamiento considerado, se podrían determinar peculiares efectos causados por la aplicación del texto constitucional ${ }^{16}$. Generalmente, por ejemplo, en los sistemas con control de constitucionalidad concentrado, las sentencias del juez constitucional tienen efectos erga omnes, mientras que en los sistemas con control difuso aquéllas tienen valor inter partes. Además, se pueden determinar peculiaridades debidas a la mayor relevancia político-social que las decisiones constitucionales suelen tener.

c) Peculiaridad de las técnicas interpretativas. ¿Es posible identificar una especificidad respecto a las técnicas interpretativas en derecho constitucional? Si por actividad interpretativa se entiende atribución de significado a un texto no parece que haya especificidad en la interpretación de la constitución ${ }^{17}$. Por el contrario, es posible identificar una especificidad res-

${ }^{15}$ Cfr. R. Guastini, Distinguendo, cit. pp. 238-241; L. Prieto Sanchís, Notas sobre la interpretación constitucional, cit., p. 176.

${ }^{16}$ Cfr. L. Prieto Sanchís, Notas sobre la interpretación constitucional, cit., pp. 185-186.

${ }^{17}$ Pero los principios, como se ha dicho, no son interpretables por esa vía; la operación dirigida a atribuir significado a estos estándars normativos no se puede limitar a un análisis lingüístico. El significado de los principios debe comprenderse en base a las concepciones morales y políticas que presuponen. Estos estándares son conceptos que remiten necesariamente a concepciones, pero en el lenguaje constitucional se dejan expresamente vagos para poder adap- 
pecto a las técnicas argumentativas en derecho constitucional. A menudo, en efecto, el juez constitucional, especialmente si se concentra en un único órgano, motiva sus decisiones en base a argumentos de principio y, otras veces, en base a argumentos de justicia. Pero estas peculiaridades de la argumentación constitucional son evidentemente de carácter contingente y no necesario. Así, de nuevo, la especificidad de la interpretación constitucional será contingente según la rigidez o flexibilidad de la constitución.

d) Especificidad del objeto. Parece que para sostener una especificidad de la interpretación constitucional en un sentido que no sea contingente es preciso sostener una especificidad del objeto «constitución». Comparando los dos modelos de constitución antes indicados se podría decir que:

A) Quien adopta el modelo descriptivo de la constitución concebida como norma, configura el documento constitucional como un documento normativo al mismo nivel que la ley. Así, al nivel de la ley, la interpretación de la constitución consiste en la atribución de significado al texto normativo: atribución de significado en el caso del juez, constatación de atribuciones de otros en el caso del observador desinteresado. En este sentido, la especificidad sería relativa a otras características peculiares del ordenamiento examinado, pero no del objeto «constitución» ${ }^{18}$. La especificidad podría estar determinada: a) por el sujeto particular, si existe, destinado a la interpretación de la constitución; b) por el uso de particulares técnicas argumentativas; c) por los peculiares efectos que pueden determinar las interpretaciones de la constitución, etc.

B) Quien adopta el modelo axiológico de la constitución concebida como norma, por el contrario, configura el objeto constitución, siempre como documento normativo, pero de tipo específico: sería tratado de forma distinta a la ley. La constitución, como se ha dicho, en el «modelo axiológico de la constitución concebida como norma» constituye una suerte de puente entre el discurso jurídico y el moral, por cuanto su interpretación no puede prescindir de valoraciones éticas. El intérprete constitucional, para atribuir significado a las disposiciones constitucionales y en primer lugar a las de principio, debe referirse a una tesis moral. El lenguaje constitucional no es interpretable con los instrumentos comúnmente utilizados para la interpretación del derecho infraconstitucional, o sea, no basta con la referencia al sen-

tarlos a concepciones diversas. Es el juez quien establece, en relación a las exigencias del caso y en cada ocasión, la mejor concepción posible de conformidad y coherencia con todo el sistema. Cfr. R. Dworkin, Taking Rights Seriously, Harvard UP, Cambridge, 1977; G. Rebuffa, Costituzionalismo e giusnaturalismo: Ronald Dworkin e la riformulazione del diritto naturale, en «Materiali per una storia della cultura giuridica», 1, 1980, pp. 209-229.

${ }^{18}$ Cfr. R. Guastini, Distinguendo, cit.; L. Prieto Sanchís, Notas sobre la interpretación constitucional, cit. 
tido común de las palabras empleadas en la disposición. De este modo, la atribución de significado conduciría, por un lado, a hallar conflictos absolutos, y por tanto llevaría a la expulsión de algunos principios; por otro, conduciría a una aplicación absoluta de algunos de ellos, produciendo decisiones axiológicamente insoportables. La diversidad del objeto «constitución»se basa, por tanto, fundamentalmente en la presencia de principios en el texto constitucional. Aquellos estándares normativos son valores morales positivizados, los cuales, para ser comprendidos requieren consideraciones morales. La adopción del modelo axiológico de constitución concebida como norma conduce, por tanto, a sostener una interpretación en algún sentido moral de la constitución. Ello presupone que el intérprete actúe comparando un modelo ideal de constitución con el modelo real e interprete a este último a la luz de las asunciones de valores derivados del primero ${ }^{19}$; obviamente todo eso se presupone siempre que tenga lugar en concreto y no en abstracto ${ }^{20}$. Claro está que mientras la axiología del intérprete cada vez sea estrechamente dependiente del caso concreto, colocándose desde el punto de vista del «buen juez», las distintas interpretaciones deberán ser argumentadas de manera tal que formen un cuadro coherente. La jerarquía axiológica instituida cambiará, de todos modos, continuamente en relación a las exigencias de justicia sustancial de cada caso concreto. El modelo axiológico de la constitución concebida como norma rechaza la concepción del siglo XVIII que considera tal documento normativo una barrera contra el poder. Este modelo, por el contrario, identifica en la constitución un objetivo, una guía, para el legislador, a la que no sólo debe adaptarse, sino que debe desarrollar; tiende, en definitiva, a configurar el documento constitucional como un instrumento capaz de modelar las relaciones sociales, y no como un mero instrumento de limitación del poder político y garantía de derechos individuales.

\section{Interpretación moral de la constitución}

La interpretación moral de la constitución, contrapuesta a una interpretación en algún sentido literal, responde a una demanda de justicia sustancial, pero, por otro lado, vuelve al derecho incierto. Obviamente, aquí se trata de una cuestión de elección de los valores que se creen prevalentes. Pero, en mi opinión, aún observando el problema desde la misma perspectiva y con el mismo objetivo, que es el histórico del constitucionalismo, es decir, la limitación del poder y la garantía de los derechos, surgen algunos problemas.

1) En primer lugar, según la doctrina neoconstitucionalista, el agente consciente del cambio constitucional, o de la interpretación evolutiva de la

\footnotetext{
${ }^{19}$ C. S. Nino, The Constitution of Deliberative Democracy, cit.

${ }^{20}$ Cfr. G. Zagrebelsky, Il diritto mite, cit., cap. VII, par. 4.
} 
constitución, es el juez constitucional. Pero también el juez ordinario en la medida en que el texto constitucional está sujeto a una interpretación dirigida a obtener normas directamente aplicables a las controversias. Esto genera un problema de compatibilidad con el equilibrio de poderes constitucionales reconocido en gran parte de las constituciones contemporáneas. Aunque ya sea claro que la aplicación del derecho no consiste en una mera subsunción del hecho en la norma y que el proceso interpretativo y decisional del juez comporta elecciones, la separación de poderes, más allá de su dimensión ideológica, ofrece un instrumento de garantía de los derechos individuales. En este modelo, en efecto, sólo el poder legislativo está legitimado para producir nuevo derecho, posición de poder al que corresponde la responsabilidad política, mientras que el poder jurisdiccional tiene tareas de garantía y tutela contra leyes que lesionen derechos. La constitución, desde este punto de vista, actúa como barrera frente a las decisiones políticas adoptadas por el legislador, limitando y circunscribiendo su competencia para producir nuevo derecho ${ }^{21}$. El poder judicial, en este cuadro, se configura como un instrumento de contrapeso del poder legislativo que anula las decisiones que traspasan los límites de tal competencia legislativa. La interpretación evolutiva de la constitución quita la tarea de las decisiones políticas de las manos del legislador y la pone «bajo tutela». Ello con el consiguiente riesgo de una concentración del poder en manos de un único cuerpo, el judicial.

2) En segundo lugar, esta interpretación de la constitución implica el hecho de que ésta no pueda ya ser asumida como norma más elevada del ordenamiento jurídico. Presupone que el texto constitucional sea interpretado a la luz de algunos principios superiores, lo cual genera el interrogante acerca de la naturaleza de tales principios supraconstitucionales ${ }^{22}$.

3) En tercer lugar, la interpretación moral de la constitución deja abierto el peligro del llamado «gobierno de los jueces» y, al menos en parte, el peligro de un gobierno de los juristas que disuelve, es cierto, el terrible peligro que en democracia se produzca la llamada «tiranía de la mayoría». "Democracia» es una palabra que designa distintas concepciones relativas a la vida política de una comunidad social. Creo, sin embargo, que el referente actual de esta palabra pueda sintetizarse en la «participación de los ciudadanos en la vida política de la comunidad», ciudadanos que se presuponen autónomos y que se autodeterminan. La regla de la mayoría, si bien

${ }^{21}$ Cfr. C. Eisenmann, La justice constitutionnelle et la haute cour constitutionnelle d'autriche (1928), Presses Unversitaires D'aix, Marseille, 1986.

${ }^{22}$ Cfr. M. Troper, La nozione di principio sovracostituzionale, en P. Comanducci y R. Guastini (a cura di), Analisi e diritto 1996, Giappichelli, Torino, 1996. 
no conceptualmente necesaria, puede sostenerse que es una necesidad empírica de funcionalidad. La locución «democracia constitucional» vehicula también distintos significados pero, también en este caso, creo que se puede identificar el actual referente conceptual de la locución en la idea de que la comunidad sea gobernada por los ciudadanos, los cuales, de algún modo, están obligados a observar un documento normativo que fija las reglas formales y sustanciales, para la discusión democrática ${ }^{23}$. El sujeto, o mejor dicho, los sujetos delegados para tomar las decisiones políticas tienen, dentro del marco constitucional, libertad para instaurar jerarquías axiológicas en relación a los objetivos socio-políticos que creen que hay que perseguir, la otra cara de la moneda de esta libertad es su responsabilidad política. Ahora bien, vista desde esta perspectiva, la interpretación moral de la constitución suscita algunas perplejidades:

a) Si la discusión intersubjetiva que caracteriza a la democracia es un valor asumido como fundamental (porque estimula la autonomía de los individuos, la participación en la vida social, etc.); si se piensa que el proceso democrático, en cuanto procedimiento dirigido a alcanzar la unanimidad de los consensos, si bien limitado por la regla de la mayoría, es más provechoso que la reflexión individual: la superioridad moral del juicio del juez, respecto a la valoración del legislador no se explica. Esto no debilita la utilidad de un control de constitucionalidad de las leyes, porque las decisiones tomadas por la mayoría podrían también siempre presentar vicios de forma y de contenido, y porque el control de constitucionalidad no es un juicio moral, sino un juicio de compatibilidad constitucional.

b) Sostener la superioridad moral del juicio de un juez respecto al juicio del legislador, significa debilitar el principio de autodeterminación de las personas colocando a los ciudadanos bajo tutela. Significa, en definitiva, confiar tareas de educación moral a la aplicación del derecho. Tampoco esto debilita la utilidad del juicio de constitucionalidad de las leyes porque han sido los propios ciudadanos, bajo los ropajes del poder constituyente, quienes han establecido una forma de auto paternalismo preveyendo en el texto constitucional una mayor tutela para algunos derechos, precisamente contra su propio intento, en la forma de una decisión de la mayoría, de lesionarlos. Tampoco en este caso el juez constitucional expresa, pues, un juicio moral.

e) Con esta perspectiva interpretativa se disuelven las razones en favor del propio proceso democrático y también las razones en favor de la persistencia de la constitución. Esta perspectiva aspira a otorgar al sujeto más

${ }^{23}$ Cfr. F. Schauer, Playing by the Rules, Clarendon Press, Oxford, 1991; C. Rustici, Quando le regole del gioco sono più dure del gioco. A proposito di «Playing by the Rules», en «Ragion pratica», 7, 1996, pp. 251-268. 
irresponsable políticamente e inamovible la tarea de reformular las decisiones políticas de valor llevadas a cabo a través del proceso democrático. Si la reflexión moral individual del juez individual es considerada superior a la discusión intersubjetiva, ¿qué sentido tiene mantener un procedimiento para la toma de decisiones colectivas de tipo democrático? La preexistencia de la constitución, entendida como documento auto-obligatorio establecido por los ciudadanos, no tendría razón de ser, ya que el juicio moral del juez individual sería siempre más justo que lo que establecen las normas constitucionales.

d) Se presenta, además, un problema fundamental para el constitucionalismo contemporáneo: ¿quién controla al controlador? La separación de poderes constituye una barrera contra la concentración de poder, pero para tutelar los derechos individuales las constituciones contemporáneas prevén algunas garantías específicas frente al poder. Así, las constituciones prevén garantías frente al poder legislativo, frente al poder ejecutivo, frente al poder judicial. Por eso, mientras el poder ejecutivo y legislativo están sujetos a controles externos al control del poder jurisdiccional, no sucede lo mismo frente a este último. Generalmente las garantías constitucionales, últimas, ofrecidas por el ordenamiento jurídico para la tutela de los derechos reconocidos en la carta fundamental son de tipo jurisdiccional. Esta técnica de tutela de los derechos se revela satisfactoria hasta que no hay algún problema de garantía frente al poder jurisdiccional, o sea, hasta que este último no se configura como un poder normativo. Pero desde el momento en que el juez es un libre intérprete, incluso moral, del derecho: ¿qué garantía tiene el ciudadano? ${ }^{24}$

e) la interpretación moral llevada a cabo por los jueces coloca a uno de los sujetos más irresponsables e inamovibles para que cumpla decisiones políticas de la comunidad.

4) En cuarto lugar, la tutela de los derechos no es ya confiada a la palabra del derecho, sino a la interpretación moral del juez. Este modo de pensar surge del postulado según el cual, dada la preocupación por la tutela de los derechos y su histórica precariedad, el terreno más seguro para su tutela sea colocarlos al nivel del discurso moral, no al nivel del discurso jurídico. Eso presupone que hay un conjunto de normas morales objetivamente válidas. Sólo así se explica esta operación. Pero la existencia de normas objetivamente válidas, al menos por ahora, representa un punto central, claro está, pero un punto central de desacuerdo. Por esta razón no parece prove-

${ }^{24}$ Cfr. R. Guastini, Le garanzie dei diritti costituzionali e la teoria dell'interpretazione, en P. Comanducci y R. Guastini (a cura di), Analisi e diritto 1990, Giappichelli, Torino, 1990, pp. 99-114. 
chosa la operación que transfiere los derechos al plano moral, quitándoles lo más genuinamente jurídico. Por ese camino sólo se crea la ilusión de su seguridad, escondiendo su intrínseca fragilidad ${ }^{25}$.

5) En quinto lugar, la aplicación dúctil ${ }^{26}$ del derecho incide directamente sobre la tutela de los derechos. La perspectiva que la doctrina neoconstitucionalista toma en consideración, quedando inamovible el objetivo histórico del constitucionalismo, parece ser unívocamente el del «buen juez dotado de buen criterio». Pero desde el punto de vista constitucional, mejor sería tal vez adoptar la perspectiva del «bad man». El derecho, en efecto, presenta dos caras: una cara de garantías y una cara de opresiones. Sostener, como hace la doctrina neoconstitucionalista, la prevalencia de la exigencia de justicia sustancial del caso concreto respecto a la exigencia de certeza de la ley, y por tanto apoyar una interpretación moral que supere una dura aplicación, conduce a sobreponer el plano moral al jurídico. Esto puede funcionar hasta que nos encontramos ante el «buen juez dotado de buen criterio», pero la dimensión garantista del derecho desaparece. Se puede poner un ejemplo. Para referirse al «viejo» sistema operativo iuspositivista, la doctrina neoconstitucionalista usa la tristemente famosa locución «la ley es la ley». Pero no advierte al lector de la ambigüedad de tal locución una vez extrapolada del contexto histórico en el que nació. «La ley es la ley» puede evocar un modo de pensar legalista, en el sentido del positivismo ideológico, para el que la justificación de una decisión jurídica, en última instancia, se descubre en la orden pronunciada por un superior, postulando la justicia de una orden por el solo hecho de provenir de un determinado sujeto. Pero, en el ámbito de una sociedad constitucional-democrático-pluralista tal locución puede leerse, también, en el sentido opuesto, es decir, en la perspectiva de un garantismo legislativo. Si se considera que la autonomía de las personas es un valor, y si se concibe el derecho como una suerte de «mal

${ }^{25}$ Algunos critican la previsión constitucional de los derechos basándose en el hecho de que tal introducción restringe su alcance, por un lado, y la previsión de un catálogo finito de derechos no garantiza «todos» los derechos reclamados por una sociedad pluralista: cfr. J. Waldrom, $A$ Rights-Based Critique of Constitutional Rights, en «Oxford Journal of Legal Studies», 13, 1993, pp. 18-5 1; contro cfr. J. J. Moreso, Derechos y justicia procesal imperfecta, ponencia inédita presentada al Il Congreso de «Ragion pratica», San Remo, Palazzo Roverizio, 12/13/14 junio 1997. Otros, como Stephen Holmes, opinan que la estrategia de la introducción de un Bill of Rights en el texto constitucional constituye una forma de auto-paternalismo (justificado), un vínculo que refuerza la democracia: cfr. S. Holmes, Vincoli costituzionali e paradosso della democrazia (1988), en G. Zagrebeisky, R P. Portinaro, J. Luther (a cura di), Il futuro della costituzione, Einaudi, 1996 pp. 167-208. Cfr., también, E. Bulygin, Sobre el status ontológico de los derechos humanos, en C. E. Alchourrón y E. Bulygin, Análisis lógico y Derecho, C. E. C., Madrid, 1991, pp. 619-625; R. Guastini, Diritti, en P. Comanducci y R. Guastini (a cura di), Analisi e diritto 1994, Giappichelli, Torino, 1994, pp. 163-174.

${ }^{26}$ Cfr. G. Zagrebelsky, Il diritto mite, cit. 
necesario» con la principal función de resolver los conflictos que se producen en la comunidad, se puede entender la locución «la ley es la ley» como garantía contra las imposiciones morales de quien sea. Si al derecho se confíala única tarea de dirimir los $\operatorname{conflictos}^{27}$, si la constitución ha establecido algunos derechos excluyéndolos del proceso democrático de discusión, si el legislador, en cuanto representante delegado de sujetos autónomos y que se autodeterminan, realiza determinadas elecciones dentro de los límites constitucionales, la afirmación «la ley es la ley» puede ser entendida como una prescripción restrictiva de la libertad interpretativa del juez, una interpretación que haciendo referencia al significado común de las palabras restringe el ámbito dispositivo de la interpretación judicial. Esto puede parecer un defecto en aquellos casos en los que la solución del derecho parece moralmente injusta, y los jueces, en efecto, en estos casos argumentan sosteniendo la presencia de lagunas: sosteniendo, por ejemplo, que «el legislador no ha tenido en cuenta este tipo de casos porque no ha pensado en ellos, pero si lo hubiera hecho habría previsto con seguridad una excepción». Pero es preciso hacer una distinción entre el caso en el que el juez está argumentando en favor de la previsión de una excepción de aquello que establece la ley (por supuesto, produciendo nuevo derecho), establece una excepción justificada bajo la consideración abstracta de la ley, según la cual ésta permanecerá también en los casos futuros, del caso en que el juez argumentando en favor de una solución distinta del caso respecto a lo que establece la ley, establece una excepción justificada bajo la consideración en concreto de la ley que no permanecerá para los casos futuros. Si, además, se entienden los derechos reconocidos en las constituciones como pretensiones, a las cuales, por tanto, corresponden una obligación por parte de otro sujeto, no se ve por qué su solidez sea mejor garantizada por el discurso moral que por el discurso jurídico. Mientras en el discurso jurídico a la atribución de un derecho, de una pretensión garantizada, le sigue la imposición de un deber a otros sujetos, y el derecho está, pues, garantizado constitucionalmente; en el discurso moral, a la atribución de un derecho no le corresponde una tutela institucionalizada, el derecho, por tanto, no constituye una pretensión garantizada. Si esto es así, si un derecho es considerado un derecho moral no jurídico, su defensa estaría a merced de las creencias éticas del juez individual, sin que el ciudadano pueda reivindicar su tutela efectiva. En el ámbito del discurso moral la tutela de los derechos sería reivindicable sólo en el caso en que se afirmase la existencia de principios morales objetivos, principios, que no se ponen en discusión. Pero, por otro lado, es un hecho que la identificación y el reconocimiento de un elenco de derechos

${ }^{27}$ Cfr. M. R. Dámaska, I volti della giustizia e del potere (1986), Il Mulino, Bologna, 1991. 
objetivos (o, al menos, universalmente aceptados) constituye sólo una reivindicación política (y ética), que no encuentra todavía un acuerdo generalizado. Si, en cambio, se opina que el derecho debe tener la función de perseguir una determinada educación moral de los ciudadanos, no considerando las objeciones a estas tesis, en el ámbito de una sociedad democrática debería ser el legislador quien desarrollara esta labor educativa y no el juez ${ }^{28}$.

(Traducción de Josep M. Vilajosana)

${ }^{28}$ En efecto, es conceptualmente compatible tanto con la democracia como con el postulado de la racionalidad de los participantes la función educativa del legislador. Un sujeto racional precisamente en virtud de tal propiedad puede delegar en otros, más cualificados que él mismo, su educación, en el mismo sentido que un estudiante está en condiciones de elegir un maestro. 